\title{
Cosmetic procedures among youths: a survey of junior college and medical students in Singapore
}

\author{
Jia Hui $\underline{\mathrm{Ng}}^{1}$, MBBS, Seth $\underline{\mathrm{Yeak}}^{2}$, MS, Natalie $\underline{\text { Phoon }^{3}}$, MS, Stephen $\underline{\mathrm{LO}}^{4}$, MD, FRCS
}

INTRODUCTION Although cosmetic procedures have become increasingly popular among the younger population in recent years, limited research on this subject has been done in the Asian context. We aimed to explore the views and knowledge regarding cosmetic procedures among junior college (JC) and medical students in Singapore.

METHODS In the first phase of the study, a cross-sectional, self-administered survey of 1,500 JC students aged 16-21 years from six JCs was conducted in 2010. The same survey was then conducted on a random sample of Year 2-5 medical students from an undergraduate medical school in 2011.

RESULTS In total, $1,164 \mathrm{JC}$ and 241 medical students responded to the surveys. There was an overall female to male ratio of $1.3: 1$. Of all the respondents, $2.5 \%$ of the $\mathrm{JC}$ students and $3.0 \%$ of the medical students admitted to having undergone cosmetic procedures. Among those who claimed to have never had cosmetic procedures done, $9.0 \%$ and $44.0 \%$ of the $\mathrm{JC}$ and medical students, respectively, responded that they would consider such procedures in the future. Those who disapproved of their peers undergoing cosmetic surgery comprised $35.0 \%$ of JC students and $56.8 \%$ of medical students. Among the JC and medical students, $52.0 \%$ and $36.1 \%$, respectively, were unaware of any risks associated with cosmetic procedures.

CONCLUSION The younger population is increasingly accepting of cosmetic procedures. However, there is a general lack of understanding of the risks associated with such procedures. Education of both the general public and medical students may help prevent potential medicolegal issues.

Keywords: attitudes, cosmetic procedures, students, survey

\section{INTRODUCTION}

In recent decades, we have seen an increase in the number of adolescents undergoing cosmetic procedures. Statistics from the American Society of Plastic Surgeons (ASPS) show that a total of 230,000 cosmetic procedures were carried out on patients younger than 18 years of age in 2011 - a startling jump from the 14,000 cosmetic procedures carried out on adolescent patients in 1996. (1) In view of this phenomenon, it has become pertinent to understand the considerations unique to this group of patients when working with them.

A Dutch study found that body image and attitudes improve as teenagers age, regardless of whether they opt to undergo cosmetic surgery. ${ }^{(2)}$ This finding highlights that adolescents, who are a particularly vulnerable group, may undergo cosmetic procedures that they may regret or find unnecessary in hindsight. It is therefore important to delve deeper into the current attitudes of adolescents toward cosmetic procedures, as well as investigate their level of understanding regarding the risks and benefits of such procedures. Crockett et al found that reality TV programmes on plastic surgery breed misconceptions regarding the risks and benefits of cosmetic procedures. ${ }^{(3)}$ In an era where Western media and influences permeate Asia, the possibility of Asian adolescents having similar misconceptions should not be ignored. Unfortunately, the majority of studies conducted on adolescents and plastic surgery were carried out in Western populations, and little is understood about Asian adolescents' attitudes toward cosmetic procedures, which are expected to differ significantly from those of their Western counterparts due to cultural differences.

Statistics from ASPS show that the majority of cosmetic procedures performed for adolescents were nonsurgical, with laser hair removal, chemical peels, microdermabrasion and botulinum toxin type $\mathrm{A}$ injection being the most common procedures. (1) Only $26 \%$ of the cosmetic procedures conducted on adolescents were surgical, with rhinoplasty, otoplasty, breast augmentation and breast reduction constituting the most common cosmetic surgical procedures. ${ }^{(1)}$ It would be of interest to find out what cosmetic procedures are most desired by Asian adolescents.

The present study aimed to explore the following aspects among a cohort consisting of junior college $(\mathrm{JC})$ and undergraduate medical students in Singapore: (a) the incidence of facial cosmetic procedures; (b) the body parts most commonly desired for alteration; (c) the extent of knowledge on the types of cosmetic procedures available and the associated potential risks; and (d) opinions regarding self, and/or their friends and family members undergoing these procedures.

\section{METHODS}

A cross-sectional survey involving 1,500 students from six JCs in Singapore was undertaken in May 2010. The survey was repeated in 2011 on 1,040 medical students from the Yong Loo Lin School of Medicine, National University of Singapore - the only undergraduate medical school in Singapore then. This study was approved by the local institutional review board.

${ }^{1}$ Department of Otorhinolaryngology, Singapore General Hospital, ${ }^{2}$ Yong Loo Lin School of Medicine, National University of Singapore, Singapore, ${ }^{3}$ Cork University Dental School and Hospital, National University of Ireland, Ireland, ${ }^{4}$ Formerly from the Department of Otorhinolaryngology, Tan Tock Seng Hospital, Singapore

Correspondence: Dr Ng Jia Hui, Resident, Department of Otorhinolaryngology, Singapore General Hospital, Outram Road, Singapore 169608. jiahui.ng@mohh.com.sg 
In the survey of JC students, paper questionnaires consisting of 21 items were handed out to the students during class time, in order to maximise the response rate. In the survey of medical students, an online questionnaire was emailed to the official corresponding email addresses of all 1,040 students, as it was not feasible to hand out paper questionnaires since the students were posted to different hospitals in Singapore.

Information collected included demographic data and any history of cosmetic procedures (facial or on other body parts). To gauge the openness of the students toward cosmetic procedures, they were asked whether they would consider cosmetic procedures themselves or approve of their peers, future children or partners undergoing cosmetic procedures, now or in the future. In order to observe the effect of crowd mentality on decision-making, the students were also asked whether it mattered if different groups of people knew that they have undergone cosmetic procedures. The questionnaire also included a question on the top three body parts that students would like changed, so as to gauge the current and future trends in cosmetic surgery among youths. To examine the students' knowledge concerning cosmetic procedures, the students were asked to name three risks associated with cosmetic procedures and to indicate, out of twelve common cosmetic procedures, the number of procedures they were aware of.

\section{RESULTS}

Of the 1,500 questionnaires handed out to JC students, 1,187 responses were collected. Of these, 23 questionnaires were excluded from the analysis due to incomplete or invalid responses, giving a response rate of $77.6 \%(1,164$ out of 1,500$)$. The female to male ratio of the 1,164 JC students was 1.3:1 and the median age was 17 (range 16-21) years. The majority of the JC respondents were of Chinese ethnicity (89.0\%), consistent with the Singapore population, which is predominantly Chinese. $82.6 \%$ of the JC respondents were Singaporeans, while the remaining came from 20 different countries.

Of the questionnaires emailed to 1,040 medical students, 241 responses were received, corresponding to a response rate of $23.2 \%$. The female to male ratio of the 241 medical students was 1.1:1 and the median year of study was Year 3 (age group 19-24 years). As with the cohort of JC students, the medical students who responded to the survey were mainly of Chinese ethnicity $(88.0 \%)$, and the majority of them were Singaporeans (98.3\%). The non-Singaporeans came from three different countries.

A total of $9(0.8 \%)$ JC students admitted to having undergone some form of facial cosmetic procedures, with female students $(n=6)$ making up the majority. None of them reported having procedures performed on other body parts. The procedures undertaken included facial lesion removal $(n=3)$, facial laser $(n=2)$, facial chemical peel $(n=2)$, rhinoplasty $(n=1)$ and blepharoplasty $(n=1)$. Among the medical students, $5(2.1 \%)$ had previously undergone cosmetic procedures. These procedures included facial melanocytic nevus removal $(n=3)$ and facial laser $(\mathrm{n}=2)$.

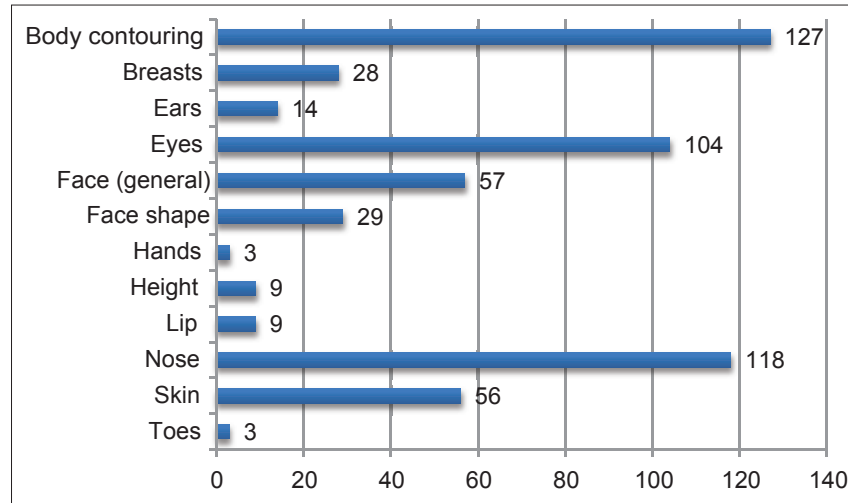

*Subjects were asked to name their top 3 desired body parts for change, if any.

Fig. 1 Bar chart shows the number of junior college students according to the specific body parts they would like altered $(n=1,164)$.

About $35.0 \%$ of the JC students approved of their peers undergoing cosmetic procedures. Among the medical students, $24.5 \%$ approved of their peers undergoing cosmetic procedures, while $32.4 \%$ of them had no opinion. More JC boys approved of their girlfriends undergoing cosmetic procedures compared to JC girls who approved of their boyfriends undergoing cosmetic procedures (22.0\% vs. $12.4 \%$ ). The opposite was observed among the 241 medical students $-17.4 \%$ of female students approved of their boyfriends undergoing cosmetic procedures, while $14.8 \%$ of male students approved of their girlfriends undergoing cosmetic procedures. When asked whether they would allow their own children to undergo cosmetic procedures in the future, $9.3 \%$ and $5.0 \%$ of the JC and medical students, respectively, indicated that they would give consent for their child to undergo cosmetic procedures as students. Among the medical students, 16.6\% were uncertain as to whether they would allow their children to undergo cosmetic procedures as students. In contrast, when asked whether they would give consent after their children have completed their education, $24.7 \%$ of the JC students and $30.7 \%$ of the medical students indicated that they would be agreeable.

Only a mere $5.0 \%$ of the JC students and $5.8 \%$ of the medical students indicated that they would consider undergoing cosmetic procedures themselves at their current age, while $9.2 \%$ of the JC students and $43.9 \%$ of the medical students indicated that they may consider it in the future. Regardless of whether the respondents admitted to considering cosmetic procedures, the top three body parts that the respondents wished to alter were the same among the JC and medical students. Among the JC students, the top three body parts were the nose $(10.1 \%)$, eyes $(8.9 \%)$ and skin (4.8\%) (Fig. 1), while among the medical students, they were the skin $(13.7 \%)$, nose (11.2\%) and eyes (9.1\%) (Fig. 2). In addition, $10.7 \%$ of the JC students and $16.6 \%$ of the medical students were keen on body contouring of areas such as the thighs, buttocks and abdomen.

When asked whether they would feel embarrassed if others were to find out that they have undergone cosmetic procedure, $28.5 \%$ and $31.5 \%$ of the JC and medical students, respectively, indicated that they would feel embarrassed if their immediate family knew, while $35.0 \%$ and $51.0 \%$ of the JC and medical students, respectively, would feel embarrassed if their close friends knew. A large percentage of the JC and medical students 


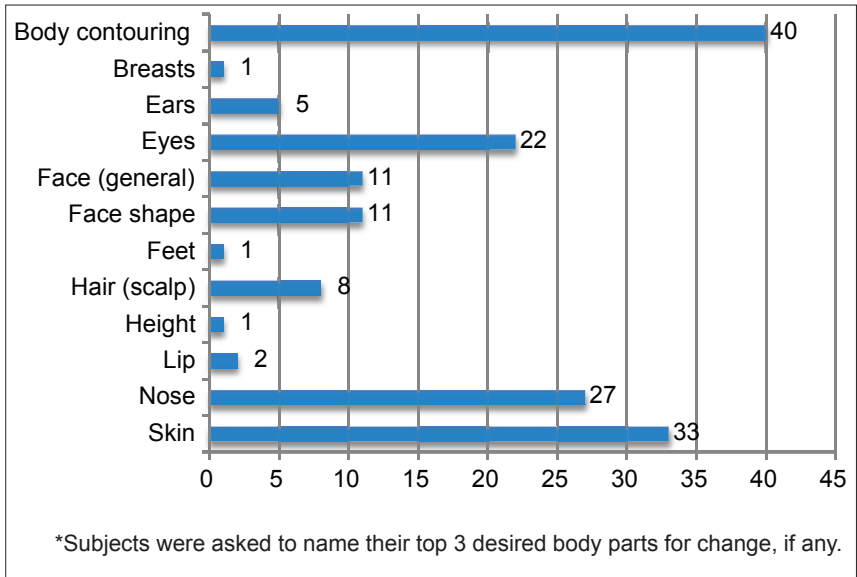

Fig. 2 Bar chart shows the number of medical students according to the specific body parts they would like altered $(n=241)$.

(50.6\% vs. $73.5 \%$ ) indicated that they would be embarrassed if people outside their family and close friends came to the knowledge that they have undergone cosmetic procedures.

A large percentage of the JC students (51.8\%) did not have any knowledge of the risks associated with cosmetic procedures. Even among the medical students, $35.7 \%$ of the students were unaware of any risks. While the remaining students believed that they knew the risks associated with cosmetic procedures and listed three risks in the questionnaire, only $22.9 \%$ of the JC students and $69.7 \%$ of the medical students managed to provide three correct associated risks.

\section{DISCUSSION}

The increasing demand for cosmetic surgery among youths in recent years could be attributed to higher disposable incomes, media portrayal and reduced stigma of cosmetic procedures. ${ }^{(4,5)}$ While reconstructive surgery to correct deformities such as cleft lip and palate has demonstrable benefits, cosmetic procedures such as liposuction and breast augmentation are much more controversial. There is no scientific evidence that cosmetic surgery improves self-esteem or confidence in the long term, although it tends to increase the patient's satisfaction with the body part that is 'fixed'. ${ }^{(6)}$ In fact, there is evidence that even without cosmetic procedures, satisfaction with appearance increases with age. ${ }^{(7)}$ Furthermore, youths who undergo cosmetic procedures may be inadequately informed of the potential complications associated with the medical or surgical procedure. Although there have been multiple studies related to the attitudes of youths on cosmetic surgery, most of these studies were conducted on Western populations. Research on this topic in non-Western settings is important, as cultural differences in attitudes toward cosmetic surgery exist. ${ }^{(8,9)}$

In the present study, only $9(0.8 \%)$ JC students and $5(2.1 \%)$ medical students admitted to having undergone cosmetic procedures. As there is a lack of national data on the number of cosmetic procedures performed on patients of different age groups in Singapore, we were unable to make comparisons with our results. In the present study, all the cosmetic procedures that the students underwent involved the face, and included skin resurfacing, facial lesion removal, blepharoplasty and rhinoplasty. Among the cosmetic procedures undergone by the 14 students,
12 procedures were minimally invasive. This mirrors the findings published in the 2011 ASPS report, which states that the number of invasive cosmetic procedures performed since 2000 have fallen by $17 \%$, while the number of minimally invasive procedures have risen by $123 \% .{ }^{(1)}$ Minimally invasive cosmetic procedures may be more popular among youths, as this population may be afraid and unprepared to 'go under the knife' to change their appearances. However, it is unknown whether youths who have undergone minimally invasive cosmetic procedures are more likely to undergo surgical cosmetic procedures later in life.

Two JC students in the present study underwent rhinoplasty $(n=1)$ and blepharoplasty $(n=1)$. As the number of students who had undergone cosmetic procedures in the present study was small, we are unable to comment whether rhinoplasty and blepharoplasty are indeed more popular than other cosmetic procedures. However, the 2010 American Academy of Facial Plastic and Reconstructive Surgery Survey found that among Asian-Americans who underwent facial plastic surgery, $44 \%$ had rhinoplasty and $47 \%$ had blepharoplasty. ${ }^{(10)}$ This may reflect the Asian desire for more Caucasian features, i.e. a higher nasal bridge and double eyelids.

Among all the participants in our study, the top three body parts that were most desirous of change involved the facial region. This is concordant with the fact that all the cosmetic procedures undergone by the students in our study also involved the face. In contrast, data from the 2011 ASPS report ${ }^{(1)}$ showed that the top three cosmetic surgical procedures performed among Western youths aged 13-19 years were rhinoplasty, male breast reduction and breast augmentation, and the top three minimally invasive cosmetic procedures performed were laser hair removal, laser skin resurfacing and laser treatment of leg veins. ${ }^{(1)}$ This difference in the types of desired cosmetic surgery between youths in Western and Asian populations may represent the effect of differences in cultural and ethnic environments.

Although we found that a large percentage of JC and medical students in Singapore were open to their peers undergoing cosmetic procedures, the majority did not intend to undergo cosmetic procedures themselves (less than $10 \%$ would consider having cosmetic procedures performed on themselves at their current age). Only $9.2 \%$ of the JC students and $43.9 \%$ of the medical students would consider plastic surgery in the future. A Norwegian study by Javo et al observed that a higher level of education was negatively correlated with an interest in cosmetic surgery. ${ }^{(4)}$ This is in sharp contrast to the results of the present study, which found that almost half of the highly educated medical students would consider cosmetic surgery in the future.

Although a substantial number of JC and medical students indicated that they would feel embarrassed if people outside of their immediate families/close friends found out that they have undergone cosmetic procedures, a large proportion also indicated that they would not feel embarrassed if their parents knew, suggesting that parental support is important in the decisionmaking process. We opine that our surveyed population is not yet ready to be open about undergoing cosmetic procedures, as many expressed embarrassment if others discovered that they have undergone such procedures. 
It is worrying that a large proportion of the students in the present study were unaware of the risks associated with cosmetic procedures. It is even more alarming that a large number of the JC students who claimed to be aware of the risks associated with cosmetic procedures listed three risks that were incorrect. This is of concern, as self-perceived knowledge of cosmetic procedures has been suggested to play a central role in influencing the choices of potential cosmetic patients. ${ }^{(3)}$ Also, previous research has shown that the general public has an inflated perception of the benefits of plastic surgery and exhibits a tendency to minimise the sense of risk of plastic surgery. ${ }^{(11)}$ This may be a more severe problem in adolescents, who have been observed to be more inclined than adults to seek instant gratification. ${ }^{(5)}$ A study by Pearl and Weston found that high school juniors developed considerable interest in undergoing cosmetic surgery simply based on the information that they had obtained from television shows and teen magazines. ${ }^{(6)}$

Although we expected a large proportion of the medical students in our study to have awareness of the risks associated with cosmetic surgery, a third of them were unaware of any associated risks. This highlights a possible loophole in Singapore's medical education, which should be addressed. Another issue that bears further exploration is the increasing interest in cosmetic surgery, which has yet to be adequately addressed by medical schools in Singapore.

A major limitation of the present study is its dependence on honest responses. Although fear of stigmatisation may have deterred some students from divulging information about the cosmetic procedures that they had undergone, we hope that this effect was minimised, as the survey was anonymous. Nevertheless, we acknowledge that the best way to obtain accurate data on cosmetic procedures performed on adolescents is the establishment of a national database that requires all cosmetic surgeons to report the procedures performed. Despite the aforementioned limitation, we opine that the present study will contribute toward a greater understanding of the attitudes of Singaporean adolescents and youths regarding cosmetic procedures.

The number of students who had undergone cosmetic procedures in our study was much lower than the number of students who indicated that they would contemplate cosmetic procedures in the future. It is unknown to what extent financial constraints contribute to this decision. Future studies investigating the role that affluence plays in the likelihood of adolescents undergoing cosmetic procedures may help shed light on this matter. It would also be useful to find out where the students had their cosmetic procedures done and whether those who were considering cosmetic procedures in the future thought that there is a need to discern between specialists and general practitioners. This has important implications on the education of both the public and general practitioners, as the practice of many new cosmetic procedures have yet to be regulated. Also, as many of these procedures are easily accessible and minimally invasive, consumers may get the false impression that the procedures are safe and complication-free. Hence, future studies should be conducted to investigate the amount of information available to consumers regarding new cosmetic treatments that are emerging with technological advancements.

In conclusion, the present study offers some insights into the attitudes and extent of knowledge on cosmetic procedures among JC and medical students in Singapore. The findings of this study suggest that the younger population is increasingly accepting of cosmetic procedures. However, there is a general lack of understanding concerning the risks associated with these procedures. Education of both the general public and medical students may help prevent potential medicolegal issues. We hope that our findings will pique interest in this area of research as well as encourage clinicians and medical educators to pay attention to the issue of adolescent cosmetic surgery in Singapore and other Asian countries

\section{REFERENCES}

1. 2011 Plastic Surgery Procedural Statistics. In: American Society for Plastic Surgeons National Clearinghouse of Plastic Surgery Procedural Statistics [online]. Available at: http://www.plasticsurgery.org. Accessed September 18, 2011.

2. Simis KJ, Hovius SE, de Beaufort ID, Verhulst FC, Koot HM. After plastic surgery: adolescent-reported appearance ratings and appearance-related burdens in patient and general population groups. Plast Reconstr Surg 2002; 109:9-17

3. Crockett RJ, Pruzinsky T, Persing JA. The influence of plastic surgery "Reality TV" on cosmetic surgery patient expectations and decision making. Plast Reconstr Surg 2007; 120:316-24.

4. Javo IM, Sørlie T. Psychosocial predictors of an interest in cosmetic surgery among young Norwegian women: a population-based study. Plast Surg Nurs 2010; 30:180-6.

5. Steinberg L. Risk-taking in adolescence: What changes, and why? In: New York Academy of Sciences 2003 meeting on adolescent brain development [online]. Available at: http://www.nyas.org/ebriefreps/main. asp?intSubsectionID320\#12. Accessed March 21, 2008.

6. Pearl A, Weston J. Attitudes of adolescents about cosmetic surgery. Ann Plast Surg 2003; 50:628-30.

7. Sarwer DB. Plastic surgery in children and adolescents. In: Thompson JK, Smolak L, eds. Body Image, Eating Disorders, and Obesity in Youth: Assessment, Prevention, and Treatment. Washington DC: APA, 2000: 341-66.

8. Swami V. Translation and validation of the Malay Acceptance of Cosmetic Surgery Scale. Body Image 2010; 7:372-5.

9. Swami V, Campana AN, Ferreira L, et al. The Acceptance of Cosmetic Surgery Scale: initial examination of its factor structure and correlates among Brazilian adults. Body Image 2011; 8:179-85.

10. 2010 International Communications Research. In: American Academy of Facial Plastic and Reconstructive Surgery Membership study. [online] Available at: http://www.aafprs.org/media/stats_polls/aafprsMedia2010. pdf. Accessed September 18, 2011.

11. Thompson JK, Smolak L. Body Image, Eating Disorders, and Obesity in Youth: Assessment, Prevention, and Treatment. Washington DC: APA; 2000: 166-341. 


\section{APPENDIX}

Attitudes of students in Singapore on cosmetic procedures

1. Medical school year (refers to the new academic year that you have started/will be going on to soon):
$\square \mathrm{M} 1$
$\square \mathrm{M} 2$
$\square \mathrm{M3}$
$\square \mathrm{M} 4$

2. Gender
$\square$ Male

\section{$\square$ Female}

3. Ethnicity
$\square$ Chinese
$\square$ Malay
$\square$ Indian
$\square$ Other

4. Nationality
$\square$ Singaporean
$\square$ Other:

5. In the past, have you had any cosmetic procedure? (If yes, please skip question 6.)
$\square$ Yes
$\square$ No
$\square$ Refrain from disclosure

If you answered yes, what cosmetic procedure did you have?

6. If you have not had any cosmetic procedures done, would you consider having cosmetic procedures for yourself now or in the future?
Now: $\square$ Yes
$\square$ No
$\square$ Maybe
Future: $\square$ Yes
$\square$ No
$\square$ Maybe

If you answered yes/maybe for any of the above, which part of your face or body would you most like to change or improve by cosmetic procedure? State three body parts in your order of preference:

7. Do you approve of students of your age undergoing cosmetic procedures?
$\square$ Yes
$\square$ No
$\square$ No opinion

8. Are you aware of any risks associated with cosmetic procedures?

$\square$ Yes

$\square$ No

If you answered yes, state three risks that you know of:

9. Would you be embarrassed about undergoing cosmetic procedures if:

(a) Your immediate family knew about it?
$\square$ Yes
$\square$ No

(b) Your close friends knew about it?
$\square$ Yes
$\square$ No

(c) People outside your immediate family and close friends knew about it?
$\square$ Yes
$\square$ No

10. Would you approve of your parent(s) undergoing cosmetic procedures if they desired to do so?
$\square$ Yes
$\square$ No
$\square$ Uncertain

11. Would you approve of your boyfriend or girlfriend undergoing cosmetic procedures if they desired to do so?
$\square$ Yes
$\square$ No
$\square$ Uncertain

$\square$ Not applicable (unattached at the moment)

12. Would you approve of your future spouse undergoing cosmetic procedures if they desired to do so?
$\square$ Yes
$\square$ No
$\square$ Uncertain

13. Would you approve of your child undergoing cosmetic procedures:

(a) While they are still students?
$\square$ Yes
$\square$ No
$\square$ Uncertain

(b) After they have completed their education?
$\square$ Yes
$\square$ No
$\square$ Uncertain

14. Prior to this survey, which of the following cosmetic procedures were you aware of?
$\square$ Abdominoplasty
$\square$ Chemical peel
$\square$ Rhytidectomy
$\square$ Blepharoplasty
$\square$ Hair removal/implant
$\square$ Bone lengthening
$\square$ Mammoplasty
$\square$ Liposuction
$\square$ Laser skin resurfacing 